2016-03-10

\title{
Chemotherapeutic Efficiency of Drugs In Vitro: Comparison of Doxorubicin Exposure in 3D and 2D Culture Matrices
}

\author{
Alan Casey \\ Technological University Dublin, alan.casey@tudublin.ie \\ Mahmoud Gargotti \\ Technological University Dublin, mahmoud.gargotti@mydit.ie \\ Franck Bonnier \\ Technological University Dublin, Franck.Bonnier@tudublin.ie
}

See next page for additional authors

Follow this and additional works at: https://arrow.tudublin.ie/biophonart

Part of the Biological and Chemical Physics Commons, and the Medicinal Chemistry and Pharmaceutics Commons

\section{Recommended Citation}

Casey, A. et al. (2016)"Chemotherapeutic efficiency of drugs in vitro: Comparison of Doxorubicin exposure in 3D and 2D culture matrices", Toxicology In Vitro, 33, 99-104 (2016). doi:10.1016/j.tiv.2016.02.022

This Article is brought to you for free and open access by the DIT Biophotonics and Imaging at ARROW@TU Dublin. It has been accepted for inclusion in Articles by an authorized administrator of ARROW@TU Dublin. For more information, please contact arrow.admin@tudublin.ie, aisling.coyne@tudublin.ie,gerard.connolly@tudublin.ie. Funder: SFI

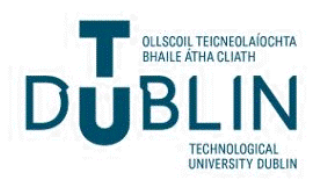




\section{Authors}

Alan Casey, Mahmoud Gargotti, Franck Bonnier, and Hugh Byrne

This article is available at ARROW@TU Dublin: https://arrow.tudublin.ie/biophonart/30 


\title{
Chemotherapeutic efficiency of drugs in vitro: Comparison of
}

\section{Doxorubicin exposure in 3D and 2D culture matrices}

\author{
A. Casey ${ }^{1,2 *}$, M. Gargotti ${ }^{1,2}$, F. Bonnier ${ }^{3}$ and H.J. Byrne ${ }^{2}$ \\ ${ }^{1}$ Nanolab Research Centre, FOCAS Research Institute, Dublin Institute of Technology, Kevin Street, \\ Dublin 8, Ireland.
}

${ }^{2}$ FOCAS Research Institute, Dublin Institute of Technology, Kevin Street, Dublin 8, Ireland.

${ }^{3}$ Faculty of Pharmacy, EA 6295 - NM/NP, Université François-Rabelais de Tours, 60 rue du Plat D'Etain, 37020 Tours Cedex 1, France

*Corresponding author : alan.casey@dit.ie

\begin{abstract}
The interest in the use of 3D matrices for in vitro analysis, with a view to increasing the relevance of in vitro studies and reducing the dependence on in vivo studies, has been growing in recent years. Cells grown in a 3D in vitro matrix environment have been reported to exhibit significantly different properties to those in a conventional 2D culture environment. However, comparison of $2 \mathrm{D}$ and $3 \mathrm{D}$ cell culture models have recently been noted to result in differing responses of cytotoxic assays, without any associated change in viability. The effect was attributed to differing conversion rates and effective concentrations of the resazurin assay in 2D and 3D environments, rather than differences in cellular metabolism. In this study, the efficacy of a chemotherapeutic agent, doxorubicin, is monitored and compared in conventional 2D and 3D collagen gel exposures of immortalized human cervical cells. Viability was monitored with the aid of the Alamar Blue assay and drug internalisation was verified using confocal microscopy. Drug uptake and retention within the collagen matrix was monitored by absorption spectroscopy. The viability studies showed apparent differences between the 2D and 3D culture systems, the differences attributed in part to the physical transition from 2D to a
\end{abstract}


$3 \mathrm{D}$ environment causing alterations to dye resazurin uptake and conversion rates. The use of 3D culture matrices has widely been interpreted to result in "reduced" toxicity or cellular "resistance" to the chemotherapeutic agent. The results of this study show that the reduced efficiency of the drug to cells grown in the $3 \mathrm{D}$ environment can be accounted for by a sequential reduction of the effective concentration of the test compound and assay. This is due to absorption within the collagen gel inducing a higher uptake of both drug and assay thereby influencing the toxic impact of the drug and conversion rate of resazurin, and. the increased effective surface area of the cell exposed to the drug and assay in the 3D environment. The effect was noted to be higher after shorter exposure periods and should be accounted for in in vitro 2D and 3D culture environment comparisons.

\section{Keywords}

Collagen I, 3D matrices, Doxorubicin, Confocal microscopy, in vitro screening 


\section{Introduction}

In recent years, directives from both the EU Directive 2010/63/EU and the US Public Law 106-545 have driven strategies to minimise the use of in vivo animal models in evaluating toxicity of materials. The strategies have been likened to the notion of a three R (Replace, Reduce and Refine) strategy, put forward by Russell and Burch (1959) in relation to testing methods. As such, significant efforts have been directed towards the development of more "realistic" in vitro testing methods which better represent in vivo conditions. One such method which has received considerable interest is the use of 3D culture systems (Breslin and O'Driscoll, 2012; Brown, 2013; Chen et al., 2015: Elliott and Yuan, 2011: Kimlin et al., 2011). The use of collagen gels or other commercially developed systems are reported to provide the cells with a microenvironment or matrix which is analogous to the extra cellular matrix (ECM) experienced by cells in vivo and is widely considered to be a better representation of the cancerous cell phenotype when compared to those grown in conventional 2D culture (Petersen et al., 1992, Prestwich, 2008, Weaver et al., 1995). The effects on cells grown in such systems compared to $2 \mathrm{D}$ systems is widely documented and it has been reported that cells can exhibit different behaviour when cultured in $3 \mathrm{D}$ when compared to $2 \mathrm{D}$, such as toxicological response, phenotype, metabolism and composition (Sowa et al.; 2010; Wang et al., 1998; Fourre et al., 2008; Harisi et al.) .

Despite being widely used, research is still on going into the effect of the additional ECM on cellular function and responses. For example, it has been shown that antibodies against B1-integrins exhibit different behaviour when tested in 2D compared to 3D models (Wang et al., 1998); induced doxorubicin-resistance by the extra cellular matrix in human osteosarcoma and HT1080 cells has been demonstrated (Fourre et al., 2008; Harisi et al., 2007); and reduced radiation induced toxicity when cells are grown in a 3D environment has also been reported (Sowa et al., 2010).

However, in an earlier study comparing the viability of cells grown in conventional $2 \mathrm{D}$ cultures to that of cells grown on collagen gel matrices, the apparent increased viability observed using the Alamar Blue cytotoxicity assay was attributed to differences in the diffusion and conversion rates of the test 
dye due to the alteration of the geometry and morphology of the test system (Bonnier et al., 2015). The study indicates that, rather than affecting a significant change in the cell metabolism, the 3D matrix alters the exposure conditions of the cells to the dye and this should be taken into account when comparing cellular exposures in 2D and 3D matrices.

The study is extended here to include exposure of the cell cultures in 2D and 3D environments to the commercially available chemotherapeutic agent Doxorubicin (DOX), chosen as a model compound, also clearly traceable due to its strong colour and fluorescence (Farhane et al., 2015). Cellular viability after exposure was evaluated with the Alamar Blue $(\mathrm{AB})$ in vitro cytotoxicity assay. The results further support earlier findings that the cell substrate and cell geometry can influence the outcome of the cell viability assay and highlight the need for adaption of existing basic protocols and indeed careful consideration in the interpretation of the results yielded.

\section{Materials and Methods}

\section{Materials.}

Doxorubicin (DOX), cell culture media, supplements and trypsinisation solution were all purchased from Sigma Aldrich Ltd. (Ireland). Alamar Blue ${ }^{\mathrm{TM}}(\mathrm{AB})$ and Collagen I from rat tail tendons (Gibco) were purchased from Biosciences (Ireland).

\section{Cell Culture}

HeLa, immortalized human cervical cells, were obtained from the ATTC (Manassas, VA, USA). Cells were cultured in DMEM supplemented with 1\% L-glutamine (200 mM) and 10\% foetal bovine serum (FBS) and maintained in a humidified atmosphere containing $5 \% \mathrm{CO}_{2}$ at $37^{\circ} \mathrm{C}$. HeLa cells were cultured until they reached approximately $75 \%$ confluency before preparing the plates for the cytotoxicity assay.

\section{Collagen substrate preparation.}


Solutions of collagen I from rat tail tendons (Gibco) were used for preparation of the collagen gels. The $5 \mathrm{mg} / \mathrm{mL}$ solution was mixed with sterile $10 \mathrm{X}$ phosphate buffered saline (PBS), sterile distilled water $\left(\mathrm{dH}_{2} \mathrm{O}\right)$ and $1 \mathrm{M} \mathrm{NaOH}$. The appropriate relative quantities of these components are determined by the final concentration $(2.5 \mathrm{mg} / \mathrm{mL})$ and volume needed. All the steps were carried out on ice to slow the gelation process. After mixing, $25 \mu \mathrm{L}$ of the solution were either placed in 96-wells-plates for the $\mathrm{AB}$ cytotoxicity assay, before incubation at $37{ }^{\circ} \mathrm{C}$ degrees in a $95 \%$ humidity incubator until a solid gel is formed, which was typically 30-40 minutes duration.

\section{Alamar Blue Viability Measurements}

For the AB assay, cells were seeded in 96 well microtiter plates (Nunc, Denmark) at a density of $1 \times 10^{5}, 5 \times 10^{4}, 4 \times 10^{4}$ and $3 \times 10^{4}$ cells/ml for 24,48 and $72 \mathrm{hr}$ exposures respectively, in $100 \mu \mathrm{l}$ of medium containing $10 \%$ FBS. At least three independent experiments were conducted with six replicate wells employed per concentration per plate in each independent experiment. After 24 hours of cell attachment, plates were washed with $100 \mu 1 /$ well PBS and treated with increasing concentrations of DOX, incubated for the required exposure time and then the viability was assessed with the aid of the Alamar blue assay as previously reported (Casey et al., 2007 and Davoren et al., 2007). Briefly, the control medium and/or the test exposure was removed, the cells were rinsed with PBS and $100 \mu$ of $\mathrm{AB}$ medium $(5 \%[\mathrm{v} / \mathrm{v}]$ solution of $\mathrm{AB})$ prepared in fresh medium (without FBS or supplements) were added to each well. The plates were then incubated for 3 hours. The AB assay measures the innate metabolic activity of cells. The oxidised indigo blue, nonfluorescing form of this chromogenic indicator dye is reduced by cellular dehydrogenases to a reduced pink fluorescent form, which can be easily monitored spectrophotometrically. Following the $3 \mathrm{~h}$ incubation period, $\mathrm{AB}$ fluorescence was quantified at the respective excitation and emission wavelength of $540 \mathrm{~nm}$ and $595 \mathrm{~nm}$. Wells containing medium and $\mathrm{AB}$ without cells were used as blanks. The mean fluorescent units for the six replicate cultures were calculated for each exposure treatment and the mean blank value was subtracted from these.

\section{Doxorubicin Exposure}


All exposure solutions were prepared fresh immediately prior to cellular exposure. A stock solution of $100 \mu \mathrm{M}$ DOX was prepared in complete DMEM medium (10\%FBS $1 \%$ L-glutamine) and then serially diluted with complete media. For exposure, cell culture medium was removed after the $24 \mathrm{hr}$ cell attachment and cells were then rinsed with $100 \mu \mathrm{L}$ PBS prior to exposure to the DOX containing medium.

\section{Microscopy.}

All images were taken on a Zeiss 510 LZSM confocal inverted microscope with an external argon ion laser $(488 \mathrm{~nm})$. Briefly, confluent cells were trypsinized and, at a concentration of $1 \times 10^{5}$ cells $/ \mathrm{ml}$, $100 \mu \mathrm{l}$ of cell suspension were seeded onto 35mm glass bottom dishes (MatTek Corporation, Ashland, MA, USA). Glass dishes were incubated for 1 hour. Following incubation, $1 \mathrm{ml}$ of cell culture media was added to cell suspensions. Dishes were re-incubated and allowed to attach for 24 hours. This protocol was altered to image the HeLa cells grown and exposed on the collagen substrates. For this, the glass bottomed culture dishes were coated with $100 \mu \mathrm{L}$ of collagen and allowed to gel as per the gel formation outlined previously. HeLa cells were then seeded and allowed to attach for a period of 24 hours. After the 24 hour attachment, both the glass and collagen substrate culture dishes were dosed with $2 \mu \mathrm{M}$ of the doxorubicin under standard exposure conditions. DOX was excited by $488 \mathrm{~nm}$ and its emission recorded at $650 \mathrm{~nm}$.

\section{Spectroscopy.}

To monitor gel uptake and retention of the DOX, acellular spectroscopic studies were performed on collagen gels. The gels were prepared in an identical manner as that in the cellular studies and dosed with media containing the required concentration as in the cellular exposure protocol. The DOX content was monitored, in situ in the well plates, with the aid of a Molecular Devices Spectramax M3 spectrometer by means of both absorbance measurements at $480 \mathrm{~nm}$ 
Statistical Analysis.

At least three independent experiments were conducted for all experiments. Test results for each assay were expressed as percentage of the unexposed control \pm standard deviation (SD). Control values were set as $100 \%$. Differences between samples and the control were evaluated using the statistical analysis package Minitab14. Statistical significant differences were set at $p<0.05$. Normality of data was confirmed with Kolmogorov-Smirnov tests to validate the assumptions found in one-way analysis of variances (ANOVA) and Dunnett's multiple comparison tests.

\section{Results}

Microscopy

Figure 1 shows the confocal microscopy images of HeLa cell cultures grown on 2D (a) and 3D matrices (c) exposed to $2 \square \mathrm{M}$ DOX medium for $24 \mathrm{hrs}$, as well as the unexposed controls on the $3 \mathrm{D}$ matrices (b).
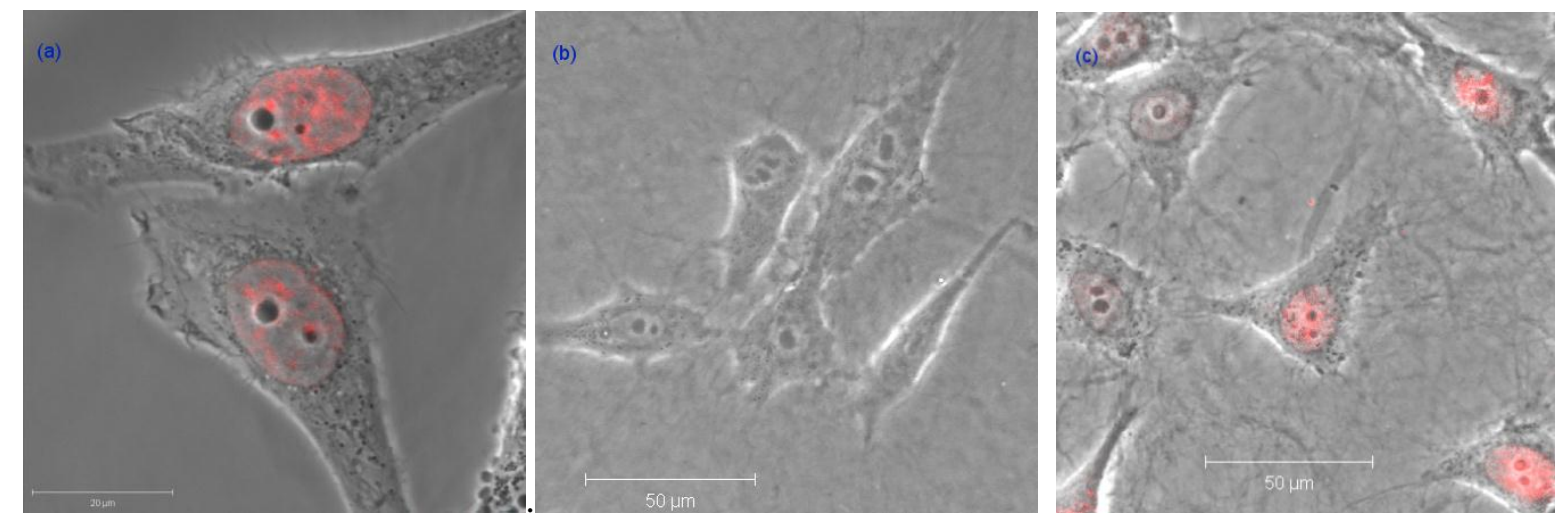

Figure 1: Confocal image of Hela cells from (a) $2 \mathrm{D}$ following $24 \mathrm{hr}$ exposure to $2 \mu \mathrm{M}$ of the doxorubicin, (b) control Hela cells grown on 3D collagen Matrix and (c) Hela cells grown on 3D matrix after $24 \mathrm{hr}$ exposure to $2 \mu \mathrm{M}$ DOX. DOX was excited by $488 \mathrm{~nm}$ and its emissions recorded at $650 \mathrm{~nm}(\mathrm{DOX})$ respectively. 
As can be seen in figure 1, no significant morphological differences were apparent between the Hela cells grown in conventional 2D (Figure 1a) when compared to that of the collagen based 3D (Figure1b), indicating that cellular growth characteristics of the Hela cells were not significantly altered by growth on the collagen substrate. Furthermore, it was verified, by monitoring the emission of the DOX by laser excitation, that the intracellular localisation site of the DOX remained unchanged in both the 2D (Figure 1a) and 3D (Figure 1c) systems and that the DOX (red) was localised primarily within the cell nucleus.

\section{Cytotoxicity}

Figure 2 shows the dose dependent cytotoxic response of HeLa cells to DOX exposure, as measured using the $\mathrm{AB}$ assay, at time points of 24,48 and $72 \mathrm{hrs}$. The continuous lines show a fit of the cytotoxic responses to Equation 1:

$$
y=\min +\frac{\max -\min }{1+\left(\frac{\mathrm{x}}{\mathrm{IC} 50}\right)^{\mathrm{Hillslope}}}
$$

where $\mathrm{IC}_{50}$ is the mean inhibitory concentration. In the case of the dose dependent curves in Figures 2 and 3, the elicited responses at the highest doses do not reach the maximum ( $0 \%$ viability). According to the description of "Operational models of pharmacological agonism" by Black and Leff (Black and Leff, 1983), the action is one of a partial agonist, and $\mathrm{IC}_{50}$ represents the concentration at which half the maximum observed affect is elicited. A clear dose dependence is observable, and, as illustrated in Table 1 , the $\mathrm{IC}_{50}$ value decreases with increasing exposure time, indicative of a progressive toxic response, as, for example, experimentally demonstrated and numerically simulated using a phenomenological rate equation approach by Maher et al. (2014), for the in vitro response to Poly (amido amide) (PAMAM) dendrimer exposure. Figure 3 shows the equivalent cytotoxic response for HeLa cells on the collagen matrices. $\mathrm{IC}_{50}$ values determined according to the fit of Equation 1 are also tabulated in Table 1, for all time points. After $24 \mathrm{hr}$ exposure, according to the AB assay, DOX is 
significantly less toxic to the HeLa cells in the 3D cell culture matrix than in the 2D culture. Notably, however, this difference is not in evidence for longer exposure times. It should also be noted that the dose dependent response at $24 \mathrm{hrs}$ on the collagen matrices has become a sharp, step like response. This response was reproducible over 6 independent measurements each using 8 replicate wells per concentration of DOX.

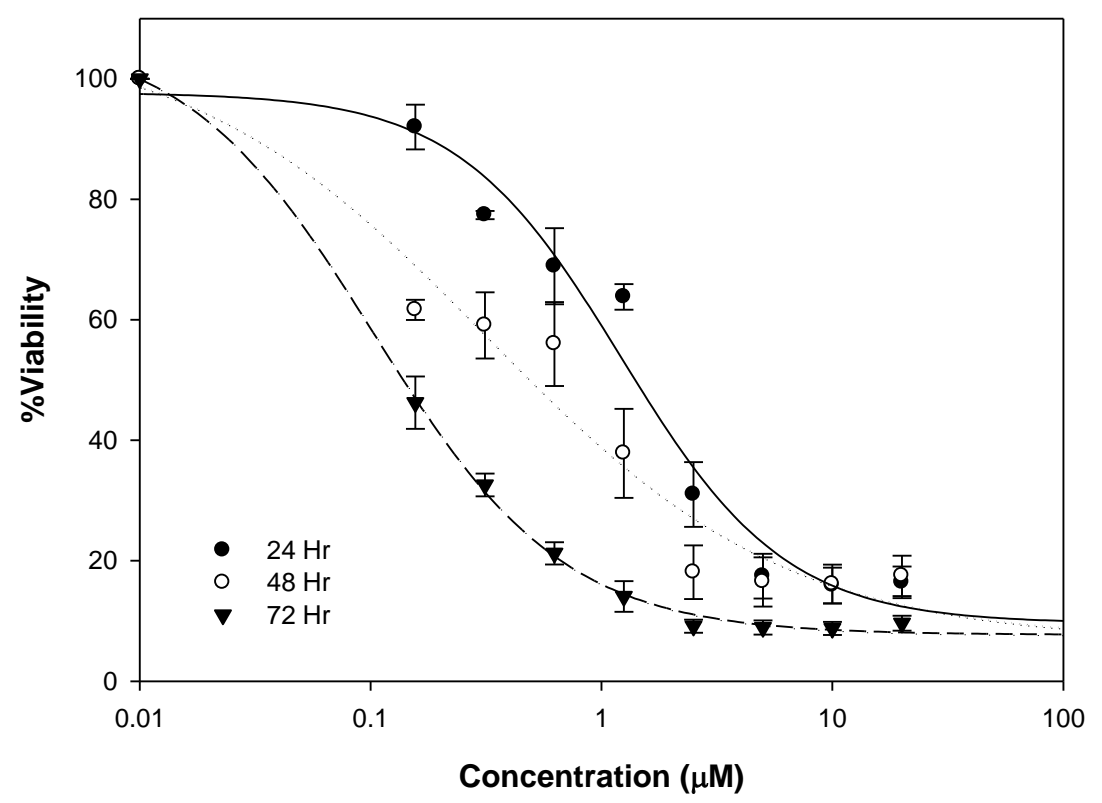

Figure 2: Alamar Blue cytotoxic response curve following 24, 48 and $72 \mathrm{hr}$ exposure of HeLa cells to Doxorubicin on a standard plate. Data is expressed as a percentage of three independent experiments \pm SD of the three individual experiments. 


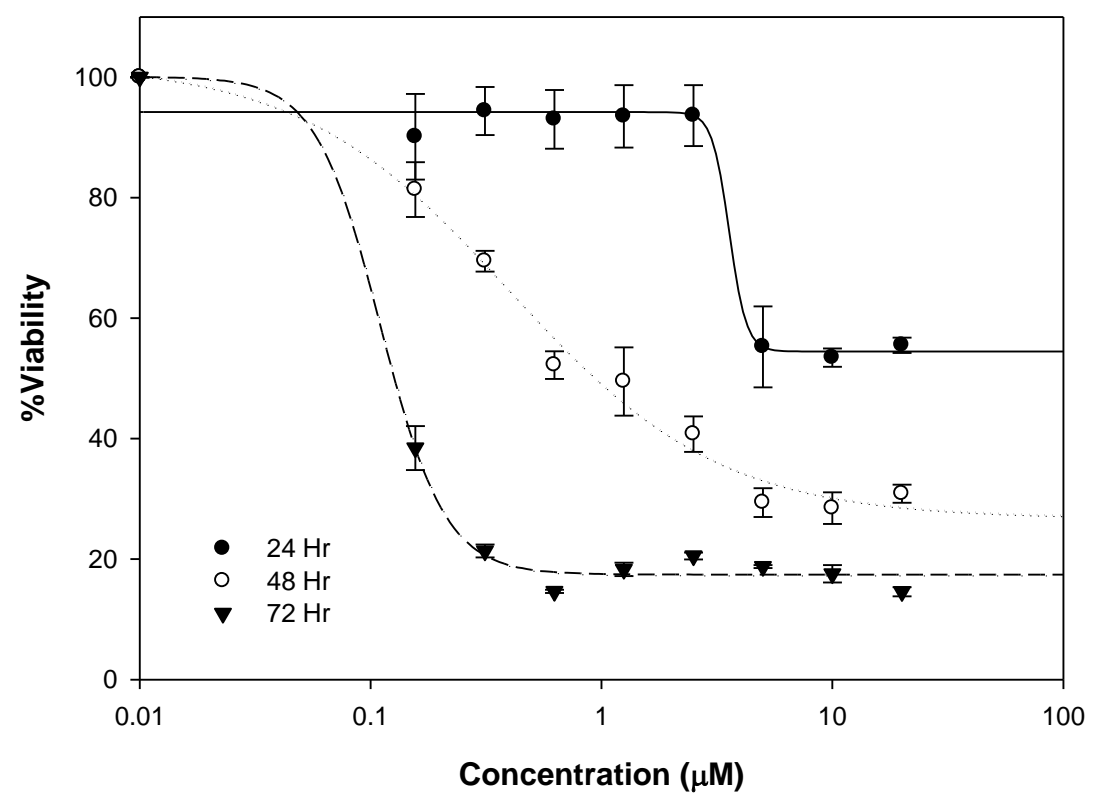

Figure 3: Alamar Blue cytotoxic response curve following 24, 48 and $72 \mathrm{hr}$ exposure of HeLa cells to Doxorubicin on a collagen coated plate. Data is expressed as a percentage of three independent experiments \pm SD of the three individual experiments.

Table 1: $\mathrm{IC}_{50}$ and Hillslope values for the fitted data values following exposure to DOX under standard culture conditions in both 2D and 3D culture substrates following 24, 48 and $72 \mathrm{Hr}$ exposure.

\begin{tabular}{|l|l|l|l|l|}
\hline Time Point $(\mathrm{Hr})$ & 2D IC $_{\mathbf{5 0}}(\boldsymbol{\mu M})$ & ${\text { 3D } \text { IC }_{\mathbf{5 0}}(\boldsymbol{\mu M})}$ & Hillslope 2D & Hillslope 3D \\
\hline $\mathbf{2 4}$ & $1.2 \pm 0.3$ & $3.6 \pm 1.33$ & $1.2 \pm 0.44$ & $11 \pm 11$ \\
\hline $\mathbf{4 8}$ & $0.3 \pm 0.22$ & $0.40 \pm 0.08$ & $0.67 \pm 0.35$ & $1.0 \pm 02.2$ \\
\hline $\mathbf{7 2}$ & $0.10 \pm 0.01$ & $0.11 \pm 0.02$ & $1.11 \pm 0.11$ & $3.1 \pm 1.33$ \\
\hline
\end{tabular}


The lower toxic response of the HeLa cells to DOX exposure after $24 \mathrm{hr}$ has been previously interpreted as an induced resistance of the cells in the 3D culture environment (Breslin and O’Driscoll, 2012; Brown, 2013; Chen et al., 2015: Elliott and Yuan, 2011: Kimlin et al., 2011). However, it has recently been demonstrated that the different cell growth environments can themselves influence the conversion rates of the cytotoxicity assay (Bonnier et al, 2015), resulting in an apparent increased viability in 3D matrices compared to $2 \mathrm{D}$ cultures. In order to establish whether similar factors affect the uptake and interaction of the DOX solution within the cells, a spectroscopic approach was employed.

Figure 4 compares the absorbance spectrum, as recorded in situ in the well plates, of the collagen gel, the cell culture medium, the cell culture medium on the collagen, $100 \mu \mathrm{M}$ DOX in cell culture medium, and $100 \mu \mathrm{M}$ DOX in cell culture medium on the collagen. The spectra are uncorrected for any background due to reflection from the well plate, which gives rise to the flat baseline of absorbance 0.05 . The cell culture medium exhibits an absorption maximum at $\sim 570 \mathrm{~nm}$, characteristic of phenol red, and a weaker maximum at $\sim 410 \mathrm{~nm}$, which derives from the FBS (Casey et al, 2008). The DOX in medium is dominated by the combination of the phenol red peak and the absorption of the DOX itself, with maxima at $\sim 500 \mathrm{~nm}$ and $480 \mathrm{~nm}$. The collagen, as expected, is transparent in the visible, only showing an onset of absorption $<400 \mathrm{~nm}$. Notably, the collagen spectrum also shows a flat baseline until this point, indicating negligible loss of light due to scattering. In contrast, the medium on collagen shows an increased baseline of absorbance $\sim 0.1$ in the transparency region, the distinctive phenol red peak at $\sim 570 \mathrm{~nm}$ is absent, and a broad absorption feature appears centred at $\sim 450 \mathrm{~nm}$. The spectrum of DOX in medium on collagen similar displays a strong scattering background in the transparency region, no evidence of the phenol red feature, and, taking into account the increased scattering baseline, a reduced absorbance of the DOX at 500nm.. 


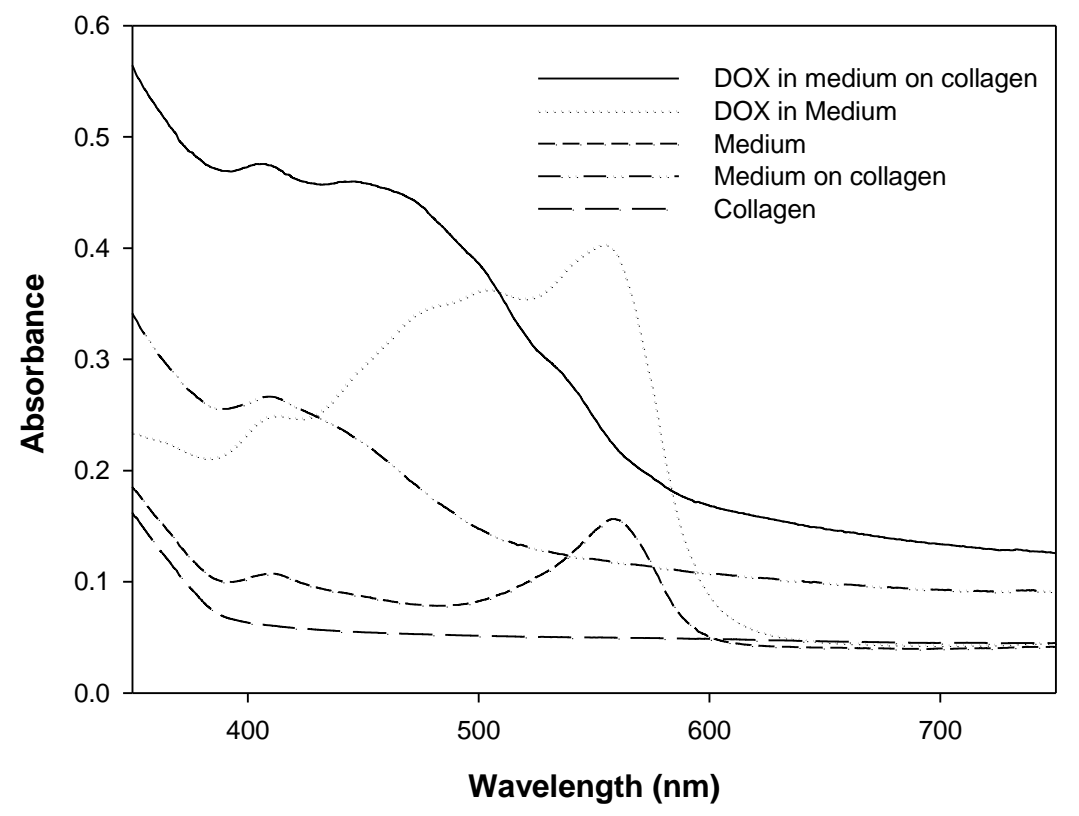

Figure 4: Absorption spectrum of cell culture medium, collagen, medium on collagen, $100 \mu \mathrm{M}$ DOX in medium, $100 \mu \mathrm{M}$ DOX in medium on collagen, recorded in situ in the well plate.

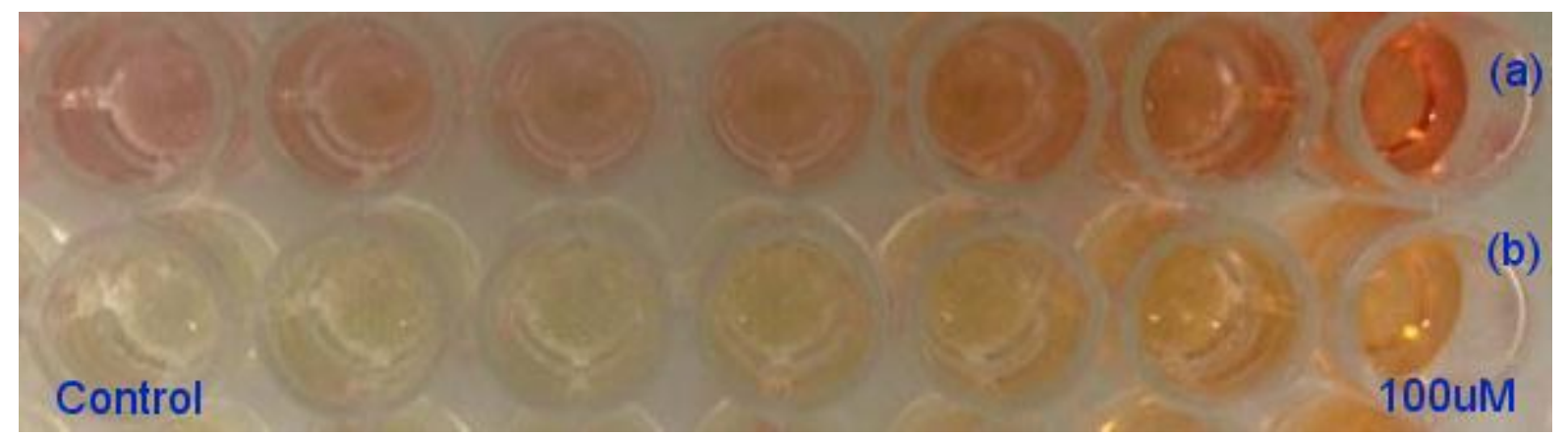

Figure 5: Photographic image of visible colour changes observed on a 96 well plate following exposure of DOX containing medium. Top row (a) is 3D exposure of $100 \mu \mathrm{M}$ DOX in medium which then underwent a serial dilution with medium. The bottom row (b) is a $2 \mathrm{D}$ exposure of a serial dilution of $100 \mu \mathrm{M}$ DOX in medium which then underwent a serial dilution with medium.

The spectral changes are well visualised in the photographic image of Figure 5, which shows a 3D exposure of a serial dilution of $100 \mu \mathrm{M}$ DOX in medium (a). The bottom row (b) shows a $2 \mathrm{D}$ exposure of a serial dilution, again of $100 \mu \mathrm{M}$ DOX in medium which was then serially diluted with fresh 
medium. The results indicate not only an interaction of the DOX with the collagen gel, but also the cell culture medium. The increased scattering background of the spectrum of the medium on collagen indicates the precipitation of medium constituents on the gel, potentially resulting in a depletion of the medium itself. The reduced DOX absorbance also indicates an interaction with the gel, and/or the precipitated constituents, potentially resulting in reduced bioavailability of the drug. Figure 6 shows the dose dependent absorbance at 480nm of DOX dispersed in the cell culture medium, with that of the supernatant extracted from a collagen gel substrate, in the absence of cells, after an exposure time of $24 \mathrm{hrs}$. The zero concentration absorbance corresponds to that of the cell culture medium, in the case of the DOX supernatant, without the contribution of the scattering from the collagen gel. Of importance to the effective dose administered to the cells, the rate of increase of DOX absorbance with concentration is considerably lower for the collagen supernatant (slope 0.0028) than the DOX medium (slope 0.0044), indicating that the effective concentration of DOX in the supernatant, which is bioavailable to the cells, is substantially lower than that of the actual dose. From the linear fits to the dose dependent absorbance, the reduction can be calculated to be a factor of $\sim 1.6$.

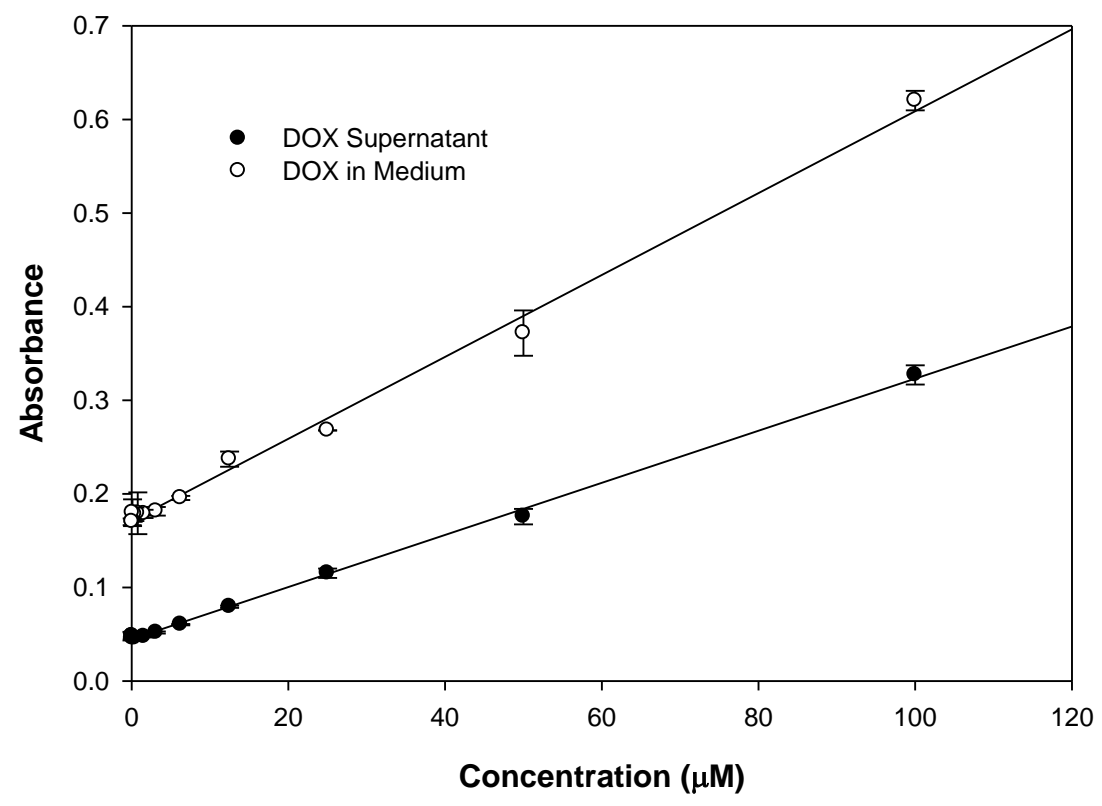

Figure 6: Dose dependent absorbance of Doxorubicin at $480 \mathrm{~nm}$ dispersed in cell culture medium (o) and that of Doxorubicin in cell culture medium after $24 \mathrm{hr}$ exposure on a collagen substrate $(\bullet)$. 
As demonstrated previously, the effective concentration of the $\mathrm{AB}$ assay is also effectively reduced by absorption by the porous collagen gel (Bonnier et al., 2015). As a further potentially confounding factor, although the gel is rinsed thoroughly between $\mathrm{DOX}$ exposure and the application of the $\mathrm{AB}$ assay, the previous exposure to the DOX may "clog" the matrix and further influence the availability of the $\mathrm{AB}$ to the cells. The absorbance (at 570nm) of the supernatant $\mathrm{AB}$ solution on the collagen gel was monitored, before and after dose dependent exposure to DOX followed by thorough rinsing. Compared to the absorbance of the initial $\mathrm{AB}$ solution, which showed an average of 0.356 , the absorbance of the $\mathrm{AB}$ supernatant of an unexposed collagen gel was found to be reduced to 0.20 after $3 \mathrm{hr}$, a factor of 1.78 . The result is consistent with that previously reported for $\mathrm{AB}$ on collagen gels (Bonnier et al., 2015). In gels which were exposed to a varying range of DOX, from $6.25 \mu \mathrm{M}$ to $100 \mu \mathrm{M}$, and subsequently thoroughly rinsed, as described in the Materials and Methods section, the absorbance of the supernatant was found to be the same, and therefore independent of DOX exposure.

\section{Discussion}

The in vitro cytotoxicity assessment of the effects of the chemotherapeutic agent DOX in cells cultures in a $2 \mathrm{D}$ compared to a $3 \mathrm{D}$ environment indicate a significant difference between the cultures and potentially an increased resistance (Fourre et al., 2008; Harisi et al., 2007) of the cells to the drug in the 3D environment. However, a similar study of the same cell line (Bonnier et al, 2015), cultured in the same two environments, demonstrated that, although the cytotoxicity study gave similar indications of reduced toxic response of the two cultures, no differences in cell population viability were apparent in a flow cytometric screening measurement. The phenomenon was explained by a combination of the absorption and therefore reduced bioavailability of the assay dye by the porous collagen matrix, and the different effective surface area of the cell exposed to the dye solution and therefore different uptake rate. The current study extends the previous study of cytotoxicity assays 
alone (Bonnier et al, 2015) to the case of the sequential exposure to the chemotherapeutic agent subsequently washed and followed by the cytotoxic assay.

The change from the 2D to the 3D cell culture environment significantly affects the profile of the dose dependent toxicity response, at all time-points, and notably at the $24 \mathrm{hr}$ time-point. The dose dependent response of toxicity assays has been comprehensively described in the treatment by Black and Leff (Black and Leff, 1983) and, more recently, the phenomenological rate equation model of Maher et al. (2014) demonstrated that the dose and time dependent responses can be reproduced by consideration of a complex cascade of events from the uptake of the external agent, to the registration of the cellular response by the cytotoxicological assay. The response profile is sensitive to the uptake rate, cellular reactions and the assay responses.

The results clearly indicate that the collagen matrix affects the effective concentration of the exposure solution, reducing it by a factor of $\sim 1.6$ which in part would contribute to the observed increase in viability previously perceived as a drug resistance (Fourre et al., 2008; Harisi et al., 2007) of cells to doxorubicin when grown in a 3D environment. Correspondingly the effective concentration of the cytotoxic assay solution is in itself reduced by a factor of 1.78 (Bonnier et al, 2015) coupled with a different effective surface area of the cell exposed to the dye solution and therefore different uptake rate; result in the reduced cytotoxic response recorded. To elicit the same degree of cell death $(50 \%)$, at $24 \mathrm{hrs}$ exposure, the actual concentration required is increased from $1.2 \pm 0.3 \mu \mathrm{M}$ to $3.6 \pm 1.3 \mu \mathrm{M}$, which can be approximately accounted for by a combination of the two factors $(1.6 / 1.78=2.85)$. The reductions of the effective concentrations of both the DOX and $\mathrm{AB}$ may account for the unusual cytotoxicity profile observed for $24 \mathrm{hr}$ exposure, in Figure 3. Notably, however, the absorption of the assay by the matrix appears to be independent of the previous DOX exposure protocol, indicating that the respective influences of the gel on the two exposure/measuring protocols they are independent of each other and can be assessed as such.

\section{Conclusions}


Although cytotoxic assessment of the effects of chemotherapeutic agents on cells in vitro may indicate a lower toxic response of cells grown on 3D matrices compared to those cultured on conventional 2D plates, interpretation of the results as a difference in cell metabolism, potentially due to increased drug resistance due to the presence of the environment which better mimics the in vivo extra cellular matrix should be made with caution. In the case of cells grown on collagen gels, the cell culture matrix shows clear interaction with the cell culture medium, itself potentially resulting in medium depletion. The bioavailability or effective concentration of the cytotoxicity assay and the chemotherapeutic agent are both affected by the absorptive nature of the matrix as is clearly evident by the variations in spectral properties and cellular responses. The study re-emphasises the need to adapt the cytotoxicity protocols commonly employed for 2D cell cultures for translation to cells grown on or in 3-D matrices.

\section{References.}

European Union - Directive 2010/63/EU of the European Parliament and of the Council of 22 September 2010

Black J. W. and Leff P. "Operational Models of Pharmacological Agonism” Proceedings of the Royal Society of London. Series B, Biological Sciences, Vol. 220, No. 1219 (Dec. 22, 1983), pp. 141162

Bonnier F., Keating M., Wróbel T.P., Majzner K., Baranska M., Garcia-Munoz A., Blanco A., Byrne H.J., 2015. Cell viability assessment using the Alamar blue assay: A comparison of 2D and 3D cell culture. Toxicology in Vitro, Volume 29, Issue 1, Pages 124-131.

Breslin S., O’Driscoll L., 2013. 3-Dimensional cell culture: the missing link in drug discovery. Drug Discovery Today 18(5-6):240-249. 
Robert A. Brown. In the beginning there were soft collagen-cell gels: towards better 3D connective tissue models? Experimental Cell Research, Volume 319, Issue 16, 1 October 2013, Pages 2460-2469

Casey A., Herzog E., Davoren M., Lyng F.M., Byrne H.J., Chambers G., 2007. Spectroscopic analysis confirms the interactions between single walled carbon nanotubes and various dyes commonly used to assess cytotoxicity. Carbon, Volume 45, Issue 7, Pages 1425-1432.

Casey A., Herzog E., Lyng F.M., Byrne H.J., Chambers G., Davoren M., 2008. Single walled carbon nanotubes induce indirect cytotoxicity by medium depletion in A549 lung. Toxicology Letters, Volume 179, Issue 2, 30 Pages 78-84.

Chen S., Nakamoto T., Kawazoe N., Chen G., 2015. Engineering multi-layered skeletal muscle tissue by using 3D microgrooved collagen scaffolds. Biomaterials, Volume 73, Pages 23-31.

Davoren M., Herzog E., Casey A., Cottineau B., Chambers G., Byrne H.J., Lyng F.M.;, 2006. Toxicology in Vitro . In vitro toxicity evaluation of single walled carbon nanotubes on human A549 lung cells. 2006, 21: 438-448 .

Elliott, N.T., Yuan, F., 2011. A review of three-dimensional in vitro tissue models for drug discovery and transport studies. Journal of Pharmacutical Science 100, 59-74.

Farhane Z., Bonnier F., Casey A., Byrne H.J., 2015. Raman micro spectroscopy for in vitro drug screening: subcellular localisation and interactions of Doxorubicin. Analyst 140(12):4212-23.

Fourre, N., Millerot-Serrurot, E., Garnotel, R., Zahm, J.M., Bonnet, N., Millot, J.M., Jeannesson, P., 2008. Extracellular matrix proteins protect human HT1080 cells against the antimigratory effect of doxorubicin. Cancer Sci 99, 1699-1705.

Harisi, R., Dudas, J., Nagy-Olah, J., Timar, F., Szendroi, M., Jeney, A., 2007. Extracellular matrix induces doxorubicin-resistance in human osteosarcoma cells by suppression of p53 function. Cancer Biol Ther 6, 1240-1246.

Kimlin, L.C., Casagrande, G., Virador, V.M., 2011. In vitro three-dimensional (3D) models in cancer research: An update. Mol Carcinog. 
Maher M.A, Naha P.C., Mukerjee S.P., Byrne H.J. 2014. Numerical simulations of in vitro nanoparticle toxicity - the case of Poly(amido amine) dendrimers" Toxicology In Vitro, 28, 14491460 .

Russell, W.M.S. and Burch, R.L., (1959). The Principles of Humane Experimental Technique, Methuen, London. ISBN 0900767782.

United States 106th Congress - Public Law 106-545, Dec. 19, 2000.

Wang F., Weaver V.M., Petersen O.W., Larabell C.A., Dedhar S., Briand P., Lupu R., Bissell M.J., 1998. Reciprocal interactions between beta1-integrin and epidermal growth factor receptor in threedimensional basement membrane breast cultures: a different perspective in epithelial biology. Proc Natl Acad Sci USA 95, 14821-14826.

Sowa M.B., Chrisler W.B., Zens K.D., Ashjian E.J., Opresko L.K., 2010. Three-dimensional culture conditions lead to decreased radiation induced cytotoxicity in human mammary epithelial cells. Mutation Research 687, 78-83.

\section{Acknowledgment}

This work was supported by Science Foundation Ireland Principle Investigator Award 11/PI/1108. 